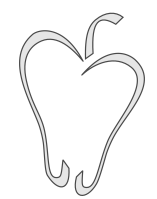

ISSN 1852-4249

\title{
Photoionization of water molecules by high energy photons
}

\author{
L. Martini, ${ }^{1 *}$ D. I. R. Boll, ${ }^{1}$ O. A. Fojón ${ }^{1 \dagger}$
}

\begin{abstract}
We theoretically study the photoionization of water molecules by high energy photon impact. We develop a model in which the final state wavefunction is given by a Coulomb continuum wavefunction with effective charges and the water molecule bound states are represented using the Moccia's monocentric wavefunctions. We obtain analytical expressions for the transition matrix element that enable the computation of cross sections by numerical quadratures. We compare our predictions for photon energies between 20 and $300 \mathrm{eV}$ with more elaborated theoretical results and experiments. We obtain a very good agreement with experiments, in particular, at enough high energies where there is a lack of elaborated results due to their high computational cost.
\end{abstract}

\section{Introduction}

Ionization of water molecules is of interest in many domains such as radiochemistry and radiobiology, as living cells are composed mostly by water. In particular, ionization of water molecules was studied in several experimental and theoretical works (see, for instance, [1-9]).

The theoretical treatment of these reactions is rather involved as the nuclear degrees of freedom of the water molecule must be taken into account as well as a proper treatment of the continuum states in the final channel of the reaction. To circumvent these inconvenients, elaborated methods were developed to obtain cross sections for photoionization of water, disregarding the vibrational and/or the rotational molecular structure. For instance, Stener et al. [2] studied the electronic transitions within the framework of the Density Func-

*E-mail: martini@ifir-conicet.gov.ar

${ }^{\dagger}$ E-mail: fojon@ifir-conicet.gov.ar

1 Laboratorio de Colisiones Atómicas, Instituto de Física Rosario (CONICET-UNR), Blvd. 27 de Febrero 210 bis, Rosario 2000, Argentina. tional Theory (DFT) with two different exchangecorrelation potentials: VWN and LB94. The first is the most common parametrization within the Local Density Approximation (LDA), whereas the second is a model potential with the correct asymptotic behavior at large distances, that is, it decays like $-1 / r$. Moreover, they considered the ground-state (GS) configuration where all electrons are kept in the calculation and the transition-state (TS) configuration where half an electron is removed from the ionized orbital. The former configuration is computationally less CPU time consuming because the ionizations of all orbitals can be obtained with the same potential, whereas in the latter, the potential depends on the orbital which is ionized and thus continuum states must be calculated separately for each state. They obtain cross sections up to approximately $70 \mathrm{eV}$. In a recent work by Gozem et al. [1] in which Dyson orbitals are used for the bound states, the final continuum states are represented by Coulomb waves describing the interaction between the active electron and the residual target. This representation is usual in high energy methods (see $[10,11]$ and references therein). The vibrational structure is considered through the Franck- 
PAPERS In Physics, vol. 9, ART. 090006 (2017) / L. Martini et al.

Condon approximation. Besides, they employ partial wave expansions to obtain matrix transition elements. In addition, they use different effective charges $Z_{\text {ef }}$ for the Coulomb waves for several molecules, such as formaldehyde, ethylene, acetylene, 1-butene, 1-butyne, methanol, and propyne. The interest was mostly the obtention of cross sections near threshold of the reactions, i.e., at low incident energies. Because of the partial wave expansions, it is expected that the above mentioned models become computationally cumbersome at higher photon energies. However, cross sections at these energies are needed to contrast with experiments and to back research on matter irradiation and related fields. Consequently, other strategies must be envisaged to obtain observables of the reaction at high impact energies.

Therefore, we adopt here a different approach and develop a Coulomb Continuum (CC) model valid at intermediate and high impact energies. We avoid the use of the Lippman-Schwinger equation or any partial wave technique that may complicate the obtention of observables at high photon energies. At first, we consider that at high enough impact energies, we can simplify the problem by assuming that the reaction occurs in a lapse much smaller than the typical vibrational and rotational times. Then, we assume that the molecule is frozen at the equilibrium configuration during the collision time. In this way, cross sections for fixed-inspace molecules can be obtained. As in most experiments, water molecules are randomly oriented, one can subsequently average over all molecular orientations to contrast the theoretical predictions. Moreover, we reduce the multielectronic problem to a monoelectronic one by considering that during the collision time one electron (the active one) is ionized and the other electrons (the passive ones) remain in their initial orbitals. In this way, our model is different from previous ones where relaxation of the target is allowed through the use of Dyson orbitals [1]. Again, our approximation is justified because the collision time is smaller than the relaxation time of the molecule. All these assumptions were employed with success in previous works (see, for instance, $[10,11]$ and references therein), rendering the problem tractable to obtain reliable cross sections. Furthermore, we describe the bound states of the water molecule by quantum chemistry methods. In particular, Moccia's wavefunctions of- fer a good description of water in terms of monocentric orbitals [12]. Even if the bound states are accessible through standard methods, the continuum states of the active electron in the final channel are still a challenge to describe in a realistic way the ionization of complex molecules. The multicenter nature of the residual target introduces complications in the theoretical descriptions. However, approximations taking into account the screening provoked by the passive electrons may be employed.

We compute cross sections within the dipole approximation for several water orbitals. We employ Moccia's wavefunctions for the bound states of the target as well as Coulomb continuum wavefunctions to describe the continuum state of the photoelectron with different effective charges to include in some way the influence of the passive electrons on the reaction. Guided by physical assumptions, we consider several effective charges to better describe the experiments. The main objective of our work is to provide reliable cross sections for photoionization of water molecules at high photon impact energies (approximately greater than $70 \mathrm{eV}$ ) where involved methods that work properly at low photon energies may turn to be prohibitive from a computational point of view.

Unless otherwise stated, atomic units are used.

\section{Theory}

\section{i. Coulomb Continuum (CC) model}

In the dipole approximation, the transition matrix amplitude for photoionization by a photon of frequency $\omega$ within the velocity gauge reads:

$$
M_{p h}^{d i p}=-i\left\langle\psi_{f}|\mathbf{e} \cdot \nabla| \psi_{i}\right\rangle,
$$

where $\psi_{i}$ denotes the electron target bound state in the initial channel and $\psi_{f}$ represents the electron continuum state in the final channel. Moreover, e is the polarization vector of the incident radiation.

Within an independent electron model in which the active electron is ionized and the passive electrons remain in their initial electronic configurations, we use the monocentric wavefunctions given by Moccia [12] to describe the different water orbitals $\psi_{i}$. This representation takes into account the electronic structure of the molecule in a basis of monocentric wavefunctions centred on the heavier atom (the oxygen atom). In addition, we work 
PAPERS In Physics, vol. 9, ART. 090006 (2017) / L. Martini et al.

with the Coulomb Continuum (CC) model in which the final continuum state of the active electron is chosen as

$$
\psi_{f}(\mathbf{r})=(2 \pi)^{-3 / 2} e^{i \mathbf{p} \cdot \mathbf{r}} N_{p} G(\mathbf{r}),
$$

where the normalization factor is given by

$$
N_{p}=e^{\pi \nu / 2} \Gamma(1+i \nu),
$$

with $\nu=Z_{e f} / p$ the Sommerfeld parameter where $Z_{\text {ef }}$ and $\mathbf{p}$ are the effective residual charge of the target and the final electron momentum respectively, and

$$
G(\mathbf{r})={ }_{1} F_{1}(-i \nu ; 1 ;-i(p r+\mathbf{p} \cdot \mathbf{r})),
$$

is the hypergeometric confluent function.

The final wavefunction of the CC model (Eq. (2)) takes into account the Coulomb interaction of the electron with the residual target in the final channel of the reaction. It was shown that this interaction must be accounted for to describe the photoionization reaction with neutral targets in a realistic way $[1,13,14]$.

By employing the Nordsieck integral, we obtain analytical expressions for the matrix transition element given by Eq. (1) (see Appendix) for each molecular orbital of the water molecule.

\section{ii. Cross sections}

Differential cross sections as a function of the photon energy and the solid angle of the ejected electron may be obtained as [15],

$$
\frac{d \sigma_{p h}(\omega)}{d \Omega_{e}}=\frac{4 \pi^{2} \alpha p}{\omega}\left|M_{p h}\right|^{2},
$$

where $\alpha$ is the fine structure constant, $\omega$ is the frequency of the incident photon, $p=|\mathbf{p}|$ is the magnitude of the asymptotic ejected electron momentum, $d \Omega_{e}=\sin \theta d \theta d \varphi$ where $\Omega_{e}$ is the solid angle of the ejection direction, and $M_{p h}$ is the matrix transition amplitude between the final and initial electronic states.

Integrating over all directions of ejection, one obtains total cross sections as a function of the photon energy,

$$
\sigma_{p h}(\omega)=\int d \Omega_{e}\left(\frac{d \sigma_{p h}(\omega)}{d \Omega_{e}}\right) .
$$

By using our analytical expression for the matrix transition element, we are able to obtain reliable cross sections at intermediate and high photon energies by standard numerical quadratures with a high degree of precision and very low CPU time.

\section{Results}

We present our cross sections for the single photoionization of randomly oriented water molecules by photons of frequency $\omega$ linearly polarized. We compare our predictions with available theoretical and experimental results. To compare with experiments, we average our cross sections over all orientations of the water molecule.

We compute our total cross sections within the dipole approximation by using the matrix transition amplitude given by Eq. (1). We have verified that the results obtained within the length and velocity gauge are almost the same in the present energy domain. In relation to the electronic final continuum states, we have considered different effective charges $Z_{\text {ef }}$ for the Coulomb function describing the photoelectron in the final channel. Gozem et al. [1] also employed effective charges for several molecules other than water.

In Fig. 1, we compare our predictions for the $1 b_{1}$, $3 a_{1}$ and $1 b_{2}$ orbitals and the total contribution with previous theoretical and experimental results. We show our calculations for different effective charges $Z_{e f}$ for the photoelectron wavefunction.

In the case of the $1 b_{1}$ orbital, the asymptotic charge value $Z_{e f}=1$ is expected to be the best choice. As a matter of fact, we can observe in Fig. 1(a) that cross sections computed with this value are in very good agreement with experiments. From a physical point of view, $Z_{e f}=1$ corresponds to the asymptotic charge of the residual target with the nuclear charges of the three centers of the water molecule completely screened by the passive electrons. Although not shown here, we have checked that effective charges less than unity give a worse agreement with experiments. Then, the ejection of an electron from the most external water orbital may be viewed as if the active electron were ejected with the hydrogen atoms acting as spectators. This is due to the non-ligand character of the $1 b_{1}$ orbital that is mostly atomic in character (in fact, no $s$-component from the $\mathrm{H}$ atoms is present 
PAPERS IN Physics, vol. 9, ART. 090006 (2017) / L. Martini et al.
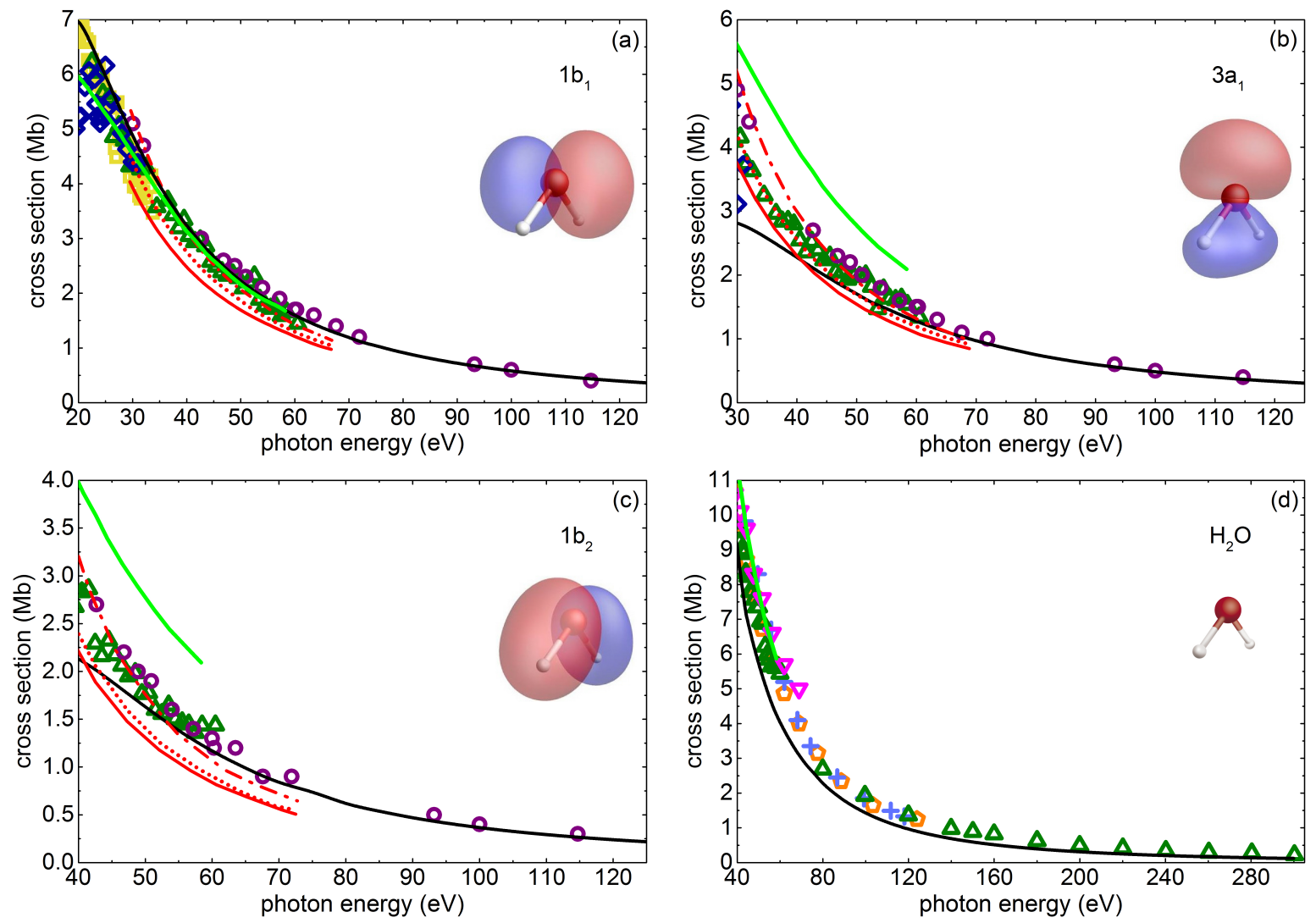

Figure 1: Total cross sections as a function of the photon energy for photoionization. Our results for (a) $1 b_{1}$ orbital with $Z_{e f}=1$, (b) $3 a_{1}$ orbital with $Z_{e f}=0.6$, (c) $1 b_{2}$ orbital with $Z_{e f}=0.1$ and (d) the total contribution. Calculations from [1] ( $(\longrightarrow)$ and from [2] for VWN GS $(\boldsymbol{-} \cdot \boldsymbol{-} \cdot)$, VWN TS (*$\Rightarrow)$ and LB94 GS $(\longrightarrow)$. Experiments: [3] $(\square),[4](\triangle),[5](\diamond),[6](\bigcirc),[7](\bullet),[8](+)$ and $[9](\nabla)$.

in the Moccia's wavefunction for this orbital). On the contrary, ejection from the $3 a_{1}$ and $1 b_{2}$ ligand orbitals necessarily involves the influence of the nuclear charges of the three nuclei of the molecule. If the active electron is emitted from the neighborhoods of the oxygen or hydrogen nuclei, it will experience an effective charge corresponding to the nuclear ones screened by the passive electrons. In the case of the hydrogen nuclei, this effective charge is necessarily less than unity. Then, an effective charge less than unity could take into account the influence of all the nuclear charges of the molecule in an averaged way. This may be related to the fact observed by Gozem et al. [1] in the description of the bound states by Dyson orbitals for several molecules, i.e., the effective charge at the centroid of such orbitals is smaller than the charge of the corresponding polyatomic cation.

Therefore, in order to describe properly ionization from the $3 a_{1}$ and $1 b_{2}$ orbitals, effective charges less than unity should be employed. It must be mentioned that in the method employed by Gozem et al. [1], the effective charge for water is taken always as $Z_{e f}=1$. This may be linked to the fact that their effective charges are related to the Mulliken population analysis in which the charges of the $\mathrm{H}$ atoms are summed onto heavy atoms. In the case of water with only one heavy atom (the oxygen atom), this leads to an effective charge $Z_{e f}=1$. In this way, their cross sections for these orbitals overestimate the measurements.

Then, we compute cross sections for those inner states of water by using adequate effective charges. The optimal effective charges could be obtained for- 
PAPERs in Physics, vol. 9, ART. 090006 (2017) / L. Martini et al.

mally by variational methods. Gozem et al. [1] develop a scheme for a one dimensional simulation with a model potential to find optimal effective charges. As a result, the optimized effective charges are very close to the ones obtained through the best fitting to the measured cross sections. It is clear that a variational procedure applied to the real three-dimensional problem may turn quite lengthy and difficult to implement due to the multicenter and multielectronic character of the molecule. However, we are not interested here in obtaining effective charges with a high numerical precision. Instead, we prefer to determine approximate effective charges and explore their physical meaning in connection with the mechanisms involved in the ionization reaction. So, we chose a simpler and practical method. After the inspection of several effective charges less than unity, we conclude that the best choices for the $3 a_{1}$ and $1 b_{2}$ orbitals are approximately $Z_{e f}=0.6$ and $Z_{e f}=0.1$, respectively.

In Fig. 1(a), we observe that our approximation for the $1 b_{1}$ orbital with $Z_{e f}=1$ gives an excellent agreement even at photon energies as low as $20 \mathrm{eV}$. We can observe that our calculations agree qualitatively well with the ones by Stener et al. [2] for photon energies greater than $30 \mathrm{eV}$ where they have almost the same slope. In particular, the best quantitative agreement with our cross sections is observed for the VWN choice with the GS configuration. In addition, we can also see that our cross sections are in very good accordance with the ones obtained with Dyson orbitals [1] for photon energies between 40 and $60 \mathrm{eV}$. However, there is some discrepancies at lower energies. Our calculations agree better with the ones given by Brion et al. [3] whereas the ones obtained with Dyson orbitals [1] are in general accordance with the ones reported by Truesdale et al. [5]. Finally, we remark that our predictions agree well with the experiments at high impact energies (around $100 \mathrm{eV}$ ) by Banna et al. [6] where there is a lack of theoretical results.

We consider now the other orbitals of water, paying attention to the use of effective charges different from unity. In Fig. 1(b), we show our results for the $3 a_{1}$ orbital together with other predictions and experiments. Our cross sections are computed with $Z_{e f}=0.6$, obtaining a very good agreement with the experiments especially at high photon energies (greater than $70 \mathrm{eV}$ ). As it can be seen, the calculations by Gozem et al. [1] with Dyson orbitals and an effective charge $Z_{e f}=1$ overestimate the measurements. On the contrary, the cross sections by Stener et al. [2] give a better description. The LB94 GS calculations tend to underestimate the experimental results whereas the VWN TS ones agree well with the measurements by Tan et al. [4] up to approximately $50 \mathrm{eV}$. The VWN GS model agrees well with the experiments by Banna et al. [6] at low energies and with the results by Tan et al. [4]; about $60-70 \mathrm{eV}$ are very close to our predictions that give the correct trend for the results by Banna et al. [6] where there exist a lack of theoretical results.

A similar situation is observed for the $1 b_{2}$ orbital in Fig. 1(c), where we show our results obtained with $Z_{e f}=0.1$, along with other theories and experiments. Again, our cross sections reproduce well the experimental results by Banna et al. [6] for photon energies greater than $70 \mathrm{eV}$ where no theoretical cross sections are available.

Finally, in Fig. 1(d), we present our total cross sections for water obtained summing the contribution of the previous relevant orbitals at the photon energies considered. We observe that the results by Gozem et al. [1] are in general agreement for experiments at low photon energies whereas our results tend to underestimate them. On the contrary, our predictions are in very good agreement with the measurements by Tan et al. [4] at high photon energy.

\section{Conclusions}

We have computed photoionization total cross sections as a function of the photon energy within the dipole approximation for photon energies from 20 $\mathrm{eV}$ and up to $300 \mathrm{eV}$. To describe the final continuum state, we employ Coulomb functions with effective charges that take into account the screening of the nuclear charges by the passive electrons. We obtain very good agreement with more elaborated previous results as well as with experiments except perhaps at low incident energies where our model is not expected to be valid. In particular, our results agree very well with measurements in the region of high enough photon energy where there is a lack of theoretical predictions. In this sense, our work aims at filling a gap providing accurate cross sections in this region. Moreover, the compu- 
PAPERS In Physics, vol. 9, ART. 090006 (2017) / L. Martini et al.

tation of cross sections with our CC model for other molecules may be performed easily. Cross sections for molecules with a heavy central atom such as $\mathrm{HF}, \mathrm{CH}_{4}, \mathrm{SiH}_{4}, \mathrm{H}_{2} \mathrm{~S}, \mathrm{HCl}, \mathrm{NH}_{3}, \mathrm{NH}_{4}^{+}, \mathrm{PH}_{3}, \mathrm{PH}_{4}^{+}$, for which Moccia's wavefunctions are available can be easily obtained. In addition, the versatility of our model enables the study of other molecules for which molecular orbitals may be safely described in terms of Slater basis. Finally, we think that our results may be useful in several domains. For instance, to study in detail the effects of ionizing radiations during its passage through living matter where our cross sections may be employed as the basic inputs to fed Monte Carlo codes to obtain the energy deposition in the biological medium.

Acknowledgements - Authors acknowledge financial support from the Agencia Nacional de Promoción Científica y Tecnológica (PICT No. 01912) and the Consejo Nacional de Investigaciones Científicas y Técnicas de la República Argentina (PIP No. 11220090101026).

\section{Appendix}

In this appendix, we present the calculations to obtain the matrix transition elements (Eq. (1)) corresponding to the Coulomb Continuum (CC) model presented in Section II.i.

Within the framework of the dipole approximation, the matrix element reads:

$$
M_{p h}^{d i p}=-i\left\langle\psi_{f}|\mathbf{e} \cdot \nabla| \psi_{i}\right\rangle .
$$

where $\psi_{i}$ is the initial electronic state, $\psi_{f}$ is the final electronic state given by Eq. (2) and where e represents the polarization vector of the incident radiation.

We employ Moccia's wavefunctions [12] to represent the initial states $\psi_{i}$ of the different water orbitals. As these functions are linear combinations of Slater orbitals, the transition matrix amplitudes for the different water orbitals are linear combinations of the transition matrix amplitudes of the Slater orbitals.

As an example, we show the calculations for the transition matrix amplitude of the simplest Slater orbital, $\chi_{1 s}$. In this case, the initial wavefunction $\psi_{i}$ for the transition matrix element (7) is given by

$$
\psi_{\chi_{1 s}}(\mathbf{r})=\frac{\alpha^{3 / 2}}{\sqrt{\pi}} e^{-\alpha r}
$$

Replacing Eqs. (8) and (2) in equation (7), one obtains:

$$
\begin{aligned}
M_{\chi_{1 s}}= & -i(2 \pi)^{-3 / 2} N_{p}^{*} \frac{\alpha^{3 / 2}}{\sqrt{\pi}} \\
& \int d^{3} r e^{-i \mathbf{p} \cdot \mathbf{r}} G^{*}(\mathbf{r}) \mathbf{e} \cdot \nabla_{\mathbf{r}}\left(e^{-\alpha r}\right) .
\end{aligned}
$$

Considering that $\nabla_{\mathbf{r}}\left(e^{-\alpha r}\right)=-\alpha e^{-\alpha r} \frac{\mathbf{r}}{r}$, one gets:

$$
\begin{aligned}
M_{\chi_{1 s}}= & i(2 \pi)^{-3 / 2} N_{p}^{*} \frac{\alpha^{5 / 2}}{\sqrt{\pi}} \\
& \int \frac{d^{3} r}{r} e^{-\alpha r-i \mathbf{p} \cdot \mathbf{r}} G^{*}(\mathbf{r})(\mathbf{e} \cdot \mathbf{r}) .
\end{aligned}
$$

Now, if we consider the polarization versor e parallel to the $z$ axis,

$$
M_{\chi_{1 s}}=i(2 \pi)^{-3 / 2} N_{p}^{*} \frac{\alpha^{5 / 2}}{\sqrt{\pi}} I_{1},
$$

where we have defined

$$
I_{1}=\int \frac{d^{3} r}{r} e^{-\alpha r-i \mathbf{p} \cdot \mathbf{r}} G^{*}(\mathbf{r}) r_{z},
$$

with $r_{z}$ the $z$-component of the position vector $\mathbf{r}$.

To solve this integral, we introduce a vector $\mathbf{v}$ parallel to the $z$ axis and we define the integral

$$
\begin{aligned}
I_{2} & =\int \frac{d^{3} r}{r} e^{-\alpha r-i \mathbf{p} \cdot \mathbf{r}} e^{i \mathbf{v} \cdot \mathbf{r}} G^{*}(\mathbf{r}) \\
& =\int \frac{d^{3} r}{r} e^{-\alpha r-i \mathbf{p} \cdot \mathbf{r}} e^{i v r_{z}} G^{*}(\mathbf{r}),
\end{aligned}
$$

which satisfies

$$
I_{1}=-i \frac{\partial}{\partial v}\left(I_{2}\right)_{v=0} .
$$

Therefore,

$$
M_{\chi_{1 s}}=(2 \pi)^{-3 / 2} N_{p}^{*} \frac{\alpha^{5 / 2}}{\sqrt{\pi}} \frac{\partial}{\partial v}\left(I_{2}\right)_{v=0} .
$$


Then, employing the Nordsieck's method [16], it is possible to obtain the integral $I_{2}$ as:

$$
\begin{aligned}
J(\lambda, \mathbf{q}, \mathbf{v})= & \int \frac{d^{3} r}{r} e^{-\lambda r+i(\mathbf{q}+\mathbf{v}-\mathbf{p}) \cdot \mathbf{r}} G^{*}(\mathbf{r}) \\
& =4 \pi f(\lambda, \mathbf{q}, \mathbf{v})
\end{aligned}
$$

where,

$$
f(\lambda, \mathbf{q}, \mathbf{v})=\frac{\left[(\mathbf{q}+\mathbf{v})^{2}+(\lambda-i p)^{2}\right]^{-i \nu}}{\left[\lambda^{2}+(\mathbf{q}+\mathbf{v}-\mathbf{p})^{2}\right]^{1-i \nu}} .
$$

Consequently,

$$
I_{2}=4 \pi f(\alpha, \mathbf{0}, \mathbf{v})
$$

and the transition matrix element:

$$
M_{\chi_{1 s}}=\frac{\sqrt{2}}{\pi} N_{p}^{*} \alpha^{5 / 2} \frac{\partial}{\partial v} f(\alpha, \mathbf{0}, \mathbf{v})_{v=0} .
$$

Finally, calculating the derivative according to equation (19), we obtain the transition matrix element corresponding to the $1 s$ Slater orbital,

$$
M_{\chi_{1 s}}=\frac{2 \sqrt{2}}{\pi} N_{p}^{*} \alpha^{5 / 2} \frac{(1-i \nu)(\mathbf{e} \cdot \mathbf{p})}{\left(\alpha^{2}+p^{2}\right)^{2}} e^{-2 \nu \operatorname{arctg}(p / \alpha)} .
$$

The matrix elements for other Slater orbitals can be computed in an analogous way. Those corresponding to the Slater orbitals $\chi_{2 s}$ and $\chi_{2 p_{0}}$ are given by, respectively,

$$
\begin{aligned}
M_{\chi_{2 s}}= & 2 \sqrt{\frac{2}{3}} \frac{1}{\pi} N_{p}^{*} \alpha^{5 / 2} \frac{(1-i \nu)(\mathbf{e} \cdot \mathbf{p})}{\left(\alpha^{2}+p^{2}\right)^{2}} \\
& {\left[\frac{3 \alpha^{2}-p^{2}-2 \nu \alpha p}{\left(\alpha^{2}+p^{2}\right)}\right] e^{-2 \nu \operatorname{arctg}(p / \alpha)}, }
\end{aligned}
$$

and,

$$
\begin{aligned}
M_{\chi_{2 p_{0}}}= & \frac{2 \sqrt{2}}{\pi} i N_{p}^{*} \alpha^{5 / 2}\left[\frac{i \nu \alpha(\alpha+i p)^{2}}{\left(\alpha^{2}+p^{2}\right)^{3}}+\right. \\
& -\frac{2 \alpha(2-i \nu)(1-i \nu)(\mathbf{e} \cdot \mathbf{p})^{2}}{\left(\alpha^{2}+p^{2}\right)^{3}}+ \\
& \left.+\frac{\nu p-\alpha}{\left(\alpha^{2}+p^{2}\right)^{2}}+\frac{\alpha(1-i \nu)}{\left(\alpha^{2}+p^{2}\right)^{2}}\right] e^{-2 \nu \operatorname{arctg}(p / \alpha)}
\end{aligned}
$$

[1] S Gozem et al, Photoelectron wave function in photoionization: Plane wave or Coulomb wave?, J. Phys. Chem. Lett. 6, 4532 (2015).

[2] M Stener, S Furlan, P Decleva, Density functional calculations of photoionization with an exchange correlation potential with the correct asymptotic behaviour, J. Phys. B: At. Mol. Opt. Phys. 33, 1081 (2000).

[3] C E Brion, F Carnovale, The absolute partial photoionization cross section for the production of the $\mathrm{X}^{2} \mathrm{~B}_{1}$ state of $\mathrm{H}_{2} \mathrm{O}^{+}$, Chem. Phys. 100, 291 (1985).

[4] K H Tan et al., Absolute oscillator strenghts (10-60 eV) for the photoabsorption photoionization and fragmentation of $\mathrm{H}_{2} \mathrm{O}$, Chem. Phys. 29, 299 (1978).

[5] C M Truesdale et al., Photoelectron angular distribution of $\mathrm{H}_{2} \mathrm{O}$, J. Chem. Phys. 76, 860 (1982).

[6] M S Banna it et al., The photoelectron spectrum of water in the 30 to $140 \mathrm{eV}$ photon energy range, J. Chem. Phys. 84, 4739 (1986).

[7] G N Haddad, J A R Samson, Total absorption and photoionization cross sections of water vapor between 100 and 1000 A, J. Chem. Phys. 84, 6623 (1986).

[8] L de Reilhac, N Damany, Photoabsorption cross section measurements of some gases from 10 to $50 \mathrm{~nm}$, J. Quant. Spectrosc. Radiat. Transfer 18, 121 (1977).

[9] E Phillips, L C Lee, D L Judge, Absolute photoabsorption cross sections for $\mathrm{H}_{2} \mathrm{O}$ and $\mathrm{D}_{2} \mathrm{O}$ from $\lambda 180$ to $790 \AA$, J. Quant. Spectrosc. Radiat. Transfer 18, 309 (1977).

[10] C A Tachino et al., Ionization of water molecules by ion beams. On the relevance of dynamic screening and the influence of the description of the initial state, J. Phys. B: At. Mol. Opt. Phys. 47, 035203 (2014).

[11] M L de Sanctis et al., Theoretical study of the ionization of liquid water from its several initial orbitals by fast electron impact, J. Phys. B: At. Mol. Opt. Phys. 48, 155201 (2015). 
Papers in Physics, vol. 9, ART. 090006 (2017) / L. Martini et al.

[12] R Moccia, One center basis set SCF MO's. III. $\mathrm{H}_{2} \mathrm{O}, \mathrm{H}_{2} \mathrm{~S}, \mathrm{HCl}$, J. Chem. Phys. 40, 2186 (1964).

[13] D I R Boll, O A Fojón, Interferences and asymmetries in laser-assisted photoionization of diatomic molecules, Phys. Rev. A 90, 053414 (2014).

[14] D I R Boll, O A Fojón, Atomic RABBITT like experiments framed as diatomic molecules,
J. Phys. B: At. Mol. Opt. Phys. 49, 185601 (2016).

[15] G L Yudin, S Patchkovskii, A D Bandrauk, Asymmetric interference in molecular photoprocesses, J. Phys. B: At. Mol. Opt. Phys. 39, 1537 (2006).

[16] A Nordsieck, Reduction of an integral in the theory of Bremsstrahlung, Phys. Rev. 93, 785 (1954). 